\title{
Short communication \\ Extended follow-up of breast cancer patients in clinic wastes time for both patients and doctors: the case for
}

J Michael Dixon ${ }^{1}$ and David A Montgomery ${ }^{2}$

1Edinburgh Breast Unit, Western General Hospital, Edinburgh EH4 2XU, UK
${ }^{2}$ Oncology, Pfizer, Walton Oaks, Dorking Road, Tadworth, Surrey KT20 7NT, UK

Corresponding author: J Michael Dixon, mike.dixon@ed.ac.uk

Published: 18 December 2008

This article is online at http://breast-cancer-research.com/content/10/S4/S7

(C) 2008 BioMed Central Ltd

\section{Current guidelines}

Numerous organizations have provided guidelines for clinical follow-up of women after they have undergone breast cancer treatment. The American Society of Clinical Oncology (ASCO) recommends clinic visits every 3 to 6 months for the first 3 years, visits every 6 to 12 months for the next 2 years, and annual visits after 5 years, with no advice on discharging patients [1]. The Canadian Steering Committee on Clinical Practice Guidelines for the Care and Treatment of Breast Cancer are more pragmatic regarding the frequency of visits, and suggests that these should be tailored to individual patient needs, but their recommendations explicitly state that follow-up should be provided indefinitely [2]. The National Institute for Health and Clinical Excellence (NICE) in the UK suggests that clinical follow-up should be limited to only 2 or 3 years [3].

There is also varying advice regarding mammographic followup. Mammography is recognized as important in the Canadian guidelines, with mammograms recommended annually [2], although the Canadian guidelines do concede that there is little high level evidence to support this practice. Similar recommendations are made by ASCO [1]. In contrast, NICE suggests that the yield of mammography is low and that local networks should decide on their own policy. The British Association of Surgical Oncology has reported that the ideal frequency of mammography has not yet been established and that mammography every 1 to 2 years should be undertaken for up to 10 years after diagnosis [4]. There is clearly disagreement between the groups that have published guidelines, but common to all of the guidelines is an emphasis on providing intensive follow-up during the first 3 to 5 years after diagnosis and treatment, with either reduced frequency of visits or discharge to the general practitioner thereafter. The bases of these guidelines are the perceptions that recurrent disease is most common in the first 3 to 5 years after
Breast Cancer Research 2008, 10(Suppl 4):S7 (doi:10.1186/bcr2167)

treatment and that clinical examination remains an important component of follow-up.

\section{Aims of follow-up}

NICE has defined the aims and objectives of follow-up: to detect and treat local recurrence; to deal with adverse effects of treatment; and to provide psychological support. Routine surveillance of metastatic disease is not recommended because data from two randomized studies revealed no improvement in outcomes for patients who undergo intensive programmes to detect and treat metastatic disease.

The GIVIO (Italian Interdisciplinary Group for Cancer Care Evaluation) investigators randomized a series of 1,320 patients with stage I, II and III unilateral primary breast cancer [5]. Patients were randomly assigned either to an intensive follow-up programme of regular bone scans, liver ultrasound, chest radiography and biochemical profile, or to a control group receiving standard care. The control group underwent regular clinical examinations and further tests as clinically indicated. Both groups had annual mammography. At a follow-up of 71 months, metastatic disease was detected in $31 \%$ of patients in the intensively observed group and in only $21 \%$ of patients in the group screened by clinical examination and mammography alone. In those in whom metastatic disease was detected, there was no significant difference in the mean time to detection of this disease (53.39 months in the intensive group versus 54.07 months in the standard care group). There was also no difference between the two groups in overall 5-year survival. The study identified no differences between the two groups in overall health, quality of life, emotional well being, body image, social functioning and satisfaction with care at $6,12,24$ and 60 months of follow-up.

The second trial, that conducted by Del Turco and colleagues [6], randomly assigned 1,234 patients with invasive unilateral 
cancers but no distant metastasis to an intensive follow-up programme of chest radiography and bone scans every 6 months, or to standard clinical follow-up. Although there were significantly more isolated intrathoracic and bone metastases detected in the intensively studied group, there was again no difference in survival. One potential criticism of both of these trials was that the duration of follow-up was short, only 5 years at the time of publication. Subsequent publication of 10-year follow-up data from the latter study, which revealed identical survival rates between those undergoing intensive follow-up and those having clinical examination and mammography alone, addressed this criticism [7].

Intensive follow-up protocols require more clinical time, are more costly and are responsible for diagnosing relatively few asymptomatic relapses. There were a large number of falsepositive scans and unnecessary investigations in the patients included in the intensively monitored group in both randomized studies, increasing anxiety in a significant number of patients [5-7].

The conclusion from these two randomized studies was that intensive follow-up programmes should be abandoned. None of the guidelines currently recommend such intensive followup. The randomized studies did use old technological investigations and were conducted at a time when therapy was limited. More effective systemic therapies are now available, and so potentially there is scope for another trial using investigations such as magnetic resonance imaging or computed tomography positron emission scanning. Such a study is currently being planned by the Eastern Cooperative Group in the USA but, outside such a study, there can be no justification for performing investigations to detect metastatic disease in asymptomatic patients during follow-up.

\section{For what purpose do patients believe that they are being followed up?}

There is clearly some confusion among patients as to why they are under follow-up. Although $68 \%$ of a group of women were aware that follow-up was aimed at detecting recurrence, only $13 \%$ believed follow-up was to detect and treat the side effects of treatment [8]. Thirty per cent thought that follow-up might have some psychological benefit [8]. There is little evidence that routine clinic visits are useful at detecting either side effects of treatment or psychological problems. Clinicians are not good at detecting psychological problems [9], and women are not keen to discuss psychological concerns in the clinic for various reasons, including embarrassment, feeling rushed and not wanting to waste the doctor's time. Studies also show that up to $71 \%$ of patients suffer distress during follow-up clinic visits, and reducing clinic visits does not appear to reduce quality of life [10]. This suggests that such visits do not contribute significantly to the detection and amelioration of physical or psychosocial problems.

\section{Patterns of relapse}

Although there is a peak of recurrence within the first 3 years, much of this is accounted for by distant disease. Moreover, recurrence continues beyond this time, and the annual rate of local recurrence is constant for at least the first 10 years [11]. The study conducted by Saphner and coworkers [11] showed that there are in fact more recurrences after the first 5 years than during the first 5 years after treatment, and so recurrence is certainly not restricted to the first 5 years. A higher early rate of relapse was observed in this study in patients with multiple positive nodes, although after year 5 the rates of local relapse were similar for all women who were node positive, regardless of the number of nodes involved.

Few studies have examined the pattern and timing of potentially treatable relapses along with the method of detection and the impact that the method of detection has on outcome. Between 1991 and 1998, a total of 1,312 patients were treated for early stage breast cancer by breast conserving surgery, axillary node sampling or clearance and postoperative radiotherapy to the breast with/without ipsilateral lymphatics in the Edinburgh Breast Unit [12]. Systemic therapy was given according to local and national guidelines. The mean age of this group of patients was 56 years, and there were 354 node-positive patients and 958 nodenegative patients. A total of 110 patients were identified who had treatable locoregional relapses: 48 in the ipsilateral axilla, of whom only three were node positive; 25 in the ipsilateral axilla; 35 in the controlateral breast; and two patients had recurrence in both breasts simultaneously. The annual rate of locoregional recurrence was constant at approximately $1.5 \%$ over the first 10 years. The mode of detection of relapse is summarized in Table 1. Overall, $51 \%$ of relapses were detected mammographically, 33.5\% were detected by the patient, $13.5 \%$ were detected by the clinician, and $2 \%$ were detected incidentally during surgery for reduction on the opposite breast. The rate of clinical detections per annual clinic visit was extremely low, at fewer than 2 per 1,000 visits, whereas the mammographic detection rate was similar to that seen within the screening programme, at 5.37 per 1,000 mammograms. Looking at patients subdivided by method of detection, the overall survival in patients who recurred locally was significantly reduced in the cohort of women whose relapse was diagnosed clinically as compared with those detected by the patient or by mammography $(P=0.0002$; log-rank test, two degrees of freedom). This difference in outcome remains significant even if patients with axillary disease are excluded $(P=0.0004)$.

It has been suggested that breast cancer follow-up can be stratified on the basis of known prognostic factors, but neither tumour size nor node status can accurately predict local recurrence or contralateral disease. Even tumour grade is a poor predictor of local recurrence. Any patient with breast cancer, when compared with a woman of the same age without cancer, has a relative risk of 3 to 4 for developing 
Table 1

\begin{tabular}{|c|c|c|c|c|c|}
\hline Site of relapse & Symptoms & Clinical finding & Mammogram & $\begin{array}{c}\text { Interval clinic } \\
\text { with symptoms }\end{array}$ & Total \\
\hline Ipsilateral breast & $4(0)$ & $4(4)$ & $20(6)$ & $11(4)$ & $39(14)$ \\
\hline Ipsilateral axilla & $5(5)$ & 9 (3) & $4(1)$ & $7(2)$ & $25(11)$ \\
\hline Ipsilateral breast and axilla & $1(1)$ & 0 & $5(0)$ & $1(0)$ & $7(1)$ \\
\hline Contralateral breast & $3(2)$ & $2(0)$ & $25(5)$ & $5(1)$ & $35(8)$ \\
\hline Ipsilateral axilla and bilateral breast & 0 & 0 & $1(0)$ & 0 & $1(0)$ \\
\hline Bilateral breast & 0 & 0 & $1(1)$ & 0 & $1(1)$ \\
\hline Total & $13(8)$ & $15(7)$ & $56(13)$ & $24(7)$ & $108(35)$ \\
\hline
\end{tabular}

Summarized are the site of relpase and the method of detection. The number of patients who subsequently died is included in parentheses. Two patients, not included in this table, had ipsilateral breast relapse diagnosed incidentally during breast reshaping procedures. Both of these patients subsequently died. Reproduced with permission from Montgomery and coworkers [12].

a new cancer in the treated breast, as well as an increased risk for developing cancer in the contralateral breast. This risk persists for at least two decades after a breast cancer diagnosis.

\section{What is optimal follow-up?}

Psychological concerns are common during follow-up, and evidence suggests that nurses detect more psychological problems than clinicians. Side effects of drug treatment are common concerns, but again these are often underestimated and unrecognized by clinicians. One solution to detect such problems is to provide patients with self-completed quality of life questionnaires, which are reliable and effective in identifying such problems. Continuing clinical input will be needed for some patients, particularly those who request revisional and reconstructive surgery, those with serious side effects from treatment, and those with signs and symptoms that suggest recurrence. Clinical input will also be needed in those who are candidates for switching to an aromatase inhibitor agent after 2 or 5 years. Agreed protocols to cope with long-term complications of treatment, including bone health, must be in place for all women. Psychological support should be available to all patients and focused when patients' needs are greatest - not only at diagnosis and during treatment, but also after treatment ends. One model that meets the aims of detecting local recurrence is annual clinical examination for 1 to 2 years and annual surveillance by mammography for 10 to 20 years or until age 80 years. Investigation of symptoms and timely communication of test results to patients and primary care is an essential component of follow-up, not only to help reduce anxiety but also to ensure that all those managing a patient are aware of the results of investigations, so that they can provide appropriate support.

What is clear is that the frequency of clinic visits advocated by ASCO is inappropriate, and these visits do not achieve their aim. The status quo cannot continue. It is time for
ASCO, NICE and others involved in establishing guidelines to utilize the large database of evidence that is currently available and abandon extended clinical follow-up programmes for patients with breast cancer. They are an ineffective use of time both for patients and for the doctors who are involved in their care.

\section{Competing interests}

The authors declare that they have no competing interests.

\section{Acknowledgement}

This article has been published as part of Breast Cancer Research Volume 10 Supplement 4, 2008: Controversies in Breast Cancer 2008. The full contents of the supplement are available online at http://breast-cancer-research.com/supplements/10/S4

\section{References}

1. Khatcheressian JL, Wolff AC, Smith TJ, Grunfeld E, Muss HB, Vogel VG, Halberg F, Somerfield MR, Davidson NE: American Society of Clinical Oncology 2006 update of the breast cancer follow-up and management guidelines in the adjuvant setting. $J$ Clin Oncol 2006, 24:5091-5097.

2. Grunfeld E, Dhesy-Thind S, Levine M, for the Steering Committee on Clinical Practice Guidelines for the Care and Treatment of Breast Cancer: Clinical practice guidelines for the care and treatment of breast cancer: follow-up after treatment for breast cancer (summary of the 2005 update). CMAJ 2005, 172:1319-1320.

3. NICE: Guidance on cancer services: improving outcomes in breast cancer - manual update. 2002 [http://www.nice.org.uk/ nicemedia/pdf/Improving_outcomes_breastcancer_manual.pdf]

4. The Association of Breast Surgery @ BASO, Royal College of Surgeons of England: Guidelines for the management of symptomatic breast disease. Eur J Surg Oncol 2005, 31(suppl 1):S1-S21.

5. The GIVO Investigators: Impact of follow-up testing on survival and health related quality of life in breast cancer patients: a multicenter randomized controlled trial. JAMA 1994, 271: 1587-1592.

6. Del Turco MR, Palli D, Carridi A, Ciatto S, Pacini P, Distante V: Intensive diagnostic follow-up after treatment of primary breast cancer. A randomized trial. National Research Council Project on Breast Cancer Follow-up. JAMA 1994, 271:1593-1597.

7. Palli D, Russo A, Saieva C Ciatto S, Del Turco MR, Distante V, Pacini P: Intensive vs clinical follow-up after treatment of primary breast cancer: 10-year update of a randomized trial. 
JAMA 1999, 281:1586.

8. Montgomery DA, Krupa K, Wilson C, Cooke TG: Patients' expectations for follow-up in breast cancer: a preliminary, questionnaire-based study. Breast 2008, 17:347-352.

9. Baildam AD, Keeling F, Thompson L, Bundred N, Hopwood P: Nurse-led surgical follow up clinics for women treated for breast cancer - a randomised controlled trial. Breast Cancer Res Treat 2004, 88(Suppl 1):S136-S137.

10. Montgomery DA, Krupa K, Cooke TG: Alternative methods of follow up in breast cancer: a systematic review of the literature. Br J Cancer 2007, 96:1625-1632.

11. Saphner T, Tormey DC, Gray R: Annual hazard rates of recurrence for breast cancer after primary therapy. J Clin Oncol $1996,14: 2738-2746$

12. Montgomery DA, Krupa K, Jack WJL, Kerr GR, Kunkler IH, Thomas J, Dixon JM: Changing pattern of the detection of locoregional relapse in breast cancer: the Edinburgh experience. Br J Cancer 2007, 96:1802-1807. 